\title{
On Optimal Quantized Non-Bayesian Quickest Change Detection With Energy Harvesting
}

\author{
Sinchan Biswas ${ }^{(\mathbb{0}}$, Student Member, IEEE, Subhrakanti Dey ${ }^{(\mathbb{1}}$, Senior Member, IEEE, \\ Steffi Knorn ${ }^{(}$, Member, IEEE, and Anders Ahlén ${ }^{(0)}$, Senior Member, IEEE
}

\begin{abstract}
In this paper, we consider a problem of decentralized non-Bayesian quickest change detection using a wireless sensor network where the sensor nodes are powered by harvested energy from the environment. The underlying random process being monitored by the sensors is subject to change in its distribution at an unknown but deterministic time point, and the sensors take samples (sensing) periodically, compute the likelihood ratio based on the distributions before and after the change, quantize it and send it to a remote fusion centre (FC) over fading channels for performing a sequential test to detect the change. Due to the unpredictable and intermittent nature of harvested energy arrivals, the sensors need to decide whether they want to sense, and at what rate they want to quantize their information before sending them to the FC, since higher quantization rates result in higher accuracy and better detection performance, at the cost of higher energy consumption. We formulate an optimal sensing and quantization rate allocation problem (in order to minimize the expected detection delay subject to false alarm rate constraint) based on the availability (at the FC) of non-causal and causal information of sensors' energy state information, and channel state information between the sensors and the FC. Motivated by the asymptotically inverse relationship between the expected detection delay (under a vanishingly small probability of false alarm) and the Kullback-Leibler (KL) divergence measure at the FC, we maximize an expected sum of the KL divergence measure over a finite horizon to obtain the optimal sensing and quantization rate allocation policy, subject to energy causality constraints at each sensor. The optimal solution is obtained using a typical dynamic programming based technique, and based on the optimal quantization rate, the optimal quantization thresholds are found by maximizing the KL information measure per slot. We also provide suboptimal threshold design policies using uniform quantization and an asymptotically optimal quantization policy for higher number of quantization bits. We provide an asymptotic approximation for the loss due to quantization of the KL measure, and also consider an alternative optimization problem with minimizing the expected sum of the inverse the KL divergence measure as the cost per time slot. Numerical results are provided comparing the various optimal and suboptimal quantization strategies for both optimization problem
\end{abstract}

Manuscript received May 27, 2019; revised August 12, 2019 and November 4, 2019; accepted December 10, 2019. Date of publication December 20, 2019; date of current version May 19, 2020. This work was supported in part by the Swedish Research Council under Grant Dnr 621-2013-5395. The associate editor coordinating the review of this article and approving it for publication was E. Ayanoglu. (Corresponding author: Subhrakanti Dey.)

Sinchan Biswas, Steffi Knorn, and Anders Ahlén are with the Division of Signals and Systems, Uppsala University, 75105 Uppsala, Sweden.

Subhrakanti Dey is with the Hamilton Institute, NUI Maynooth, W23 F2H6 Maynooth, Ireland, and also with the Division of Signals and Systems, Uppsala University, 75105 Uppsala, Sweden (e-mail: subhra.dey@mu.ie).

Digital Object Identifier 10.1109/TGCN.2019.2961113 formulations, illustrating the comparative performance of these strategies at different regimes of quantization rates.

Index Terms-Energy harvesting, sensor networks, decentralized change-point detection, quantization.

\section{INTRODUCTION}

W IRELESS sensor networks (WSN) has become an enabling technology in diverse fields of applications such as industrial process monitoring [1], mobile robots and autonomous vehicles [2], smart grid monitoring [3] and environmental data gathering [4]. In many such applications, the sensors are placed in remote or hazardous locations. Periodic replacement of batteries for such sensors can be difficult, and often expensive in other applications such as smart homes. In order to mitigate this issue, the possibility of harvesting renewable energy from the environment in order to power the sensors has been investigated in the literature. In this paradigm, the sensors are equipped with a finite sized rechargeable battery or an energy storage device, and they are capable of harvesting energy from ambient sources like temperature gradients, vibrations, wind and solar energy and storing it in the battery for future use in sensing, processing, communication and decision making. The downside of such an arrangement is the inherent random and unpredictable nature of energy harvesting processes. Coupled with the fact that each sensor has access to limited energy storage, the problem of finding optimal energy allocation for sensing, processing and information transmission poses significant challenges in practice.

Substantial amounts of research have been directed toward communication systems capable of harvesting energy from the renewable sources recently [5]. The channel capacity for such systems has been analyzed for both unlimited and limited battery scenarios [6], [7]. These results have been further extended to multiuser communication systems as well. The capacity region and optimal power scheduling problems in multiple access channels [8], interference channels [9] and relay channels [10] has been studied in the domain of multiuser networks. Simultaneous Wireless Information and power transfer in such networks has also been studied recently [11].

Energy harvesting wireless sensor networks have also received considerable attention from the research community, e.g., in the form of optimal energy management policies [12], 
power allocation policies [13], and energy efficient transmission policies [14]. Significant research has also been devoted to the domain of wireless powered crowd sensing, which focuses on joint power transfer, sensing, compression and transmission process [15]. Furthermore, the problem of optimal node deployment and energy provisioning has been studied in [16]. One important task of WSNs deployed in structural health monitoring or surveillance applications for example, is to detect changes in the underlying distribution of the observation signal. In a parametric setting (where modeling assumptions regarding the distribution before and after the change can be made), this can be accomplished either by different classical detection techniques [17] or sequential detection techniques, e.g., quickest change detection [18]. The quickest change detection method has been applied to detect anomalies or predicting failures in diverse application domains, e.g., seismic event detection, health monitoring, and detecting vacant radio spectrum. This detection technique can either be applied by the individual sensors locally or by the fusion centre (FC) after collecting information from the sensors [19]. In a parallel line of investigation, there has been recent research works investigating the change detection problem in a non-parametric and online streaming settings [20], [21]. In the current paper, however, we focus on a quickest sequential change detection problem with multiple energy harvesting sensors in a parametric setting, where the sensors send quantized log-likelihood information to the fusion centre for decision making under the assumption that the distributions before and after the change are known.

The quickest change detection problem focuses on detecting sudden changes in the distribution of the probability density function of a stochastic process while minimizing the detection delay, subject to a constraint on the false alarm rate. Here detection delay is defined as the amount of time required to detect the change point after the actual occurrence of such an event. In classical literature, there exists two ways of analyzing such events. The first one is the Bayesian formulation, which assumes the unknown change point to be drawn from a specific probability distribution [22]. The other formulation is non-Bayesian, which instead typically assumes that the change point is unknown but deterministic in nature [23], [24]. Assuming that $\mathbf{X}_{k}$ denotes the vector of sensor observations at the $k^{t h}$ time instant, the standard non-Bayesian formulation consists of a sequence of such observations $\left\{\mathbf{X}_{k}, k=1,2, \ldots\right\}$ with a change point $\lambda$. This implies that the sequence of observations before the change point, i.e., $\mathbf{X}_{1}, \mathbf{X}_{2}, \ldots, \mathbf{X}_{\lambda-1}$ is generated from the distribution $f_{0}$ whereas after the change the corresponding sequence $\mathbf{X}_{\lambda}, \mathbf{X}_{\lambda+1}, \ldots$ is generated from the distribution $f_{1}$.

In standard settings over a slotted time interval, the sensors in a wireless sensor network are able to observe the signal of interest during every time slot in the quickest change detection framework. But this is not guaranteed in the harvesting based scenario under consideration, due to the fact that each sensor is powered by ambient energy harvested from its surroundings and the stochastic nature of energy availability. The quickest change detection problem with energy constraints has been studied for both the non-Bayesian [25] and the Bayesian [26] framework in centralized settings. Decentralized quickest change detection has been studied without energy constraints in [27], [28]. To the best of our knowledge, there has not been any research in the domain of non-Bayesian quickest change detection with energy harvesting in a decentralized setting where individual sensors quantize their observation/likelihood ratio and forward this information to an FC for making a global decision. The importance of such a problem lies in the fact that at every time instant, each sensor has to make a decision regarding sensing the observation signal and sending its quantized observation to the FC for performing the quickest change detection based on the available energy in its battery. In this decentralized setting, finding an optimal quantization strategy (including rate allocation and selection of quantization thresholds) at each individual sensor is essential because the performance of the detection process is sensitive to the accuracy of the information collated at the FC. Higher number of quantization bits ensures that the information sent to the FC is more accurate, but also consumes more energy. Furthermore, each sensor's participation in the sensing process is limited by the available energy in its battery [denoted as the energy state information (ESI)] at the start of each time slot.

In this paper, we consider the problem of designing an optimal quantization and sensing policy for a decentralized quickest change detection setup where the sensors quantize their log-likelihood ratios corresponding to the distributions after and before the change, respectively, and transmit this quantized information to the FC over fading channels. The corresponding channel state information (CSI) (assumed to be known at the FC) determines the amount of energy required to transmit each quantized observation to the FC with a certain probability of error. Thus, at every time instant all sensors have to decide whether to sense the observation signal and how to quantize it, for sending it to the FC based on the amount of energy the sensors have at their disposal and the corresponding channel gain. Upon reception of the quantized measurements, the FC employs a Cumulative Sum (CUSUM) based change detection algorithm, where the cumulative sum of the total log-likelihood ratio of the quantized measurements received at the FC is compared against a threshold to detect a change. It is well known that when the probability distributions of the sensed random variables before and after the change are known (parametric setting), CUSUM based on monotone likelihood ratio quantizers (MLRQ) at the sensors achieves asymptotic optimality, in that, it minimizes the worst case average detection delay subject to a false alarm probability that approaches zero [19]. In fact, the expected detection delay is asymptotically (as the false alarm rate goes to zero) inversely proportional to the Kullback-Leibler (KL) divergence measure available at the FC, which motivates us to obtain an optimal sensing and quantization policy at the sensors, by maximizing the expected sum of an appropriate KL divergence measure over a finite horizon, subject to energy causality constraints at all sensors, given either non-causal or causal information about the available energy state information at the batteries and the sensors' channel gains.

The main contributions of our paper are as follows. 
1) The problem of minimizing the average detection delay is formulated for the non-Bayesian decentralized quickest change detection in a fading WSN over a finite time horizon where each sensor is capable of harvesting energy from the surroundings, where the energy harvesting model is far more general than a binary model used in [25]. The strategy is to maximize an expected sum of the KL divergence measure at the FC over a finite horizon for both non-causal and causal CSI and ESI.

2) The optimal policies regarding sensing decision and number of quantization bits are determined using a dynamic programming (DP) algorithm for both the non-causal and causal cases.

3) A system of nonlinear equations is formulated for deriving the optimal quantization thresholds, when the number of quantization bits are obtained from DP.

4) In the case of a large number of quantization bits, an asymptotically optimal policy is obtained by simplifying the above mentioned system of equations using the Mean Value Theorem (MVT).

5) Using asymptotic quantization theory for non-quadratic distortion measures, an asymptotic approximation is obtained for the difference between the quantized and unquantized KL divergence measure. The performances of the optimal, a heuristic uniform and the MVT based asymptotic quantizers are compared with respect to this theoretically obtained asymptotic expression.

6) The optimal and heuristic uniform quantization strategies are compared by their average detection delay for both the non-causal and causal scenario via numerical simulation results. They indicate that the optimal strategy significantly outperforms its uniform counterpart when the number of quantization bits is low. The uniform quantization policy performs closer to the optimal policy when the number of quantization bits becomes higher.

7) An alternative optimization problem is proposed for minimizing the detection delay for the same framework, by minimizing the average sum of the inverse of the KL divergence measure over a finite horizon.

8) Finally, the solutions to the two optimization problems are compared by their performance in terms of detection delay. The corresponding plots show that the alternative optimization problem performs better compared to its original counterpart, but only at the expense of an unfavourable property regarding the probability of false alarm rates.

It should be noted that some of these results stated above were partially presented in our conference paper [29]. The additional contributions of the current work compared to [29] consist of the asymptotic analysis for quantization of KL divergence measure, and the development of the asymptotic quantization policy based on MVT, the formulation of the alternative optimization problem and its performance comparison, along with additional numerical results.

The rest of the paper is organized as follows. We describe the System Model in Section II. The expected sum of $\mathrm{KL}$ divergence based optimization problem is formulated

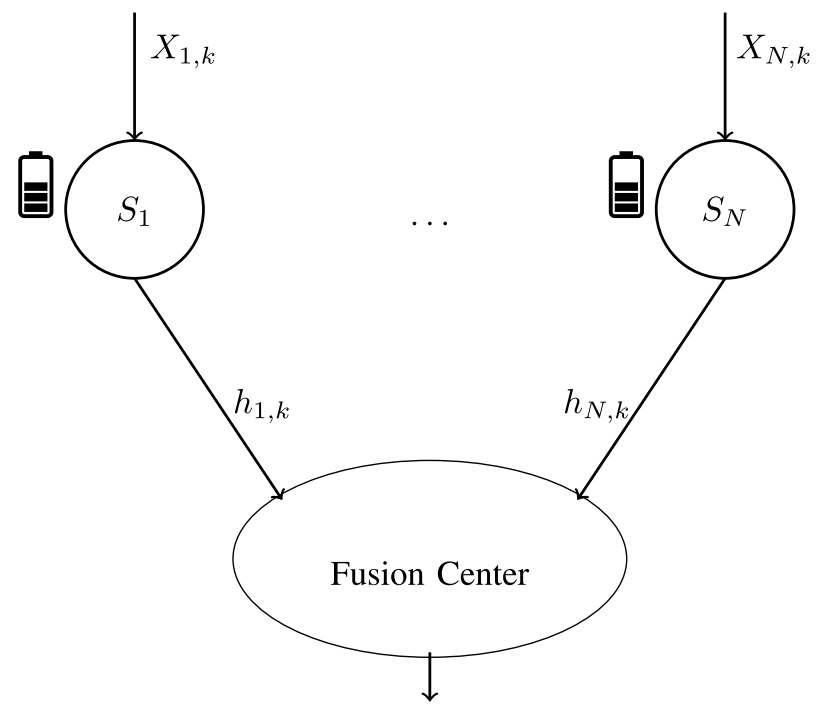

Change Point Detection

Fig. 1. Quickest change detection with distributed sensors.

in Section III. We derive the system of nonlinear equations for determining the optimal quantization thresholds in Section IV. We also introduce the MVT based asymptotic optimal quantizer and uniform quantizer in the same section. In Section V, we derive the asymptotic analytic bounds for the KL divergence measure for the optimal quantization strategy. In Section VI, we focus on finding the optimal sensing decision and quantization strategy for both non-causal and causal CSI scenario using DP. An alternative optimization problem for minimizing the detection delay is also proposed in same section. Simulation results are presented in Section VII, followed by conclusions and future extensions in Section VIII.

\section{SySTEM MODEL}

As shown in Fig. 1, the system model consists of $N$ wireless sensors and a FC for centralized decision making. We assume that each sensor is equipped with a re-chargeable battery/energy storage device of finite capacity and they are capable of harvesting energy from the surroundings. We also assume that the time is slotted and the observation signal is monitored by each sensor for $M$ time slots. During the $k^{\text {th }}$ time slot, the $i^{\text {th }}$ sensor decides to either sense or abstain from sensing based on the available energy in its battery. The corresponding binary sensing decision parameter is denoted by $\nu_{i, k}$ for $1 \leq i \leq N, 1 \leq k \leq M$, where $\nu_{i, k} \in\{0,1\}$, and 1 (or 0 ) represents the decision to sense (or not), respectively.

If the $i^{\text {th }}$ sensor senses during the $k^{\text {th }}$ time slot, i.e., $\nu_{i, k}=1$, it obtains the sample $X_{i, k}$ of the observation signal. For the system model under investigation, it is assumed that the observation signal is drawn from one of the two probability distribution functions $f_{0}$ or $f_{1}$, depending on whether it is received before or after the change point $\lambda$, respectively. As per usual, we assume that the observations signals $\left\{X_{i, k}\right\}$ are independent and identically distributed (i.i.d.) over time and across all the sensors, both before and after the change. Thus, 
we formulate the following hypothesis testing problem:

$$
\begin{aligned}
& \mathcal{H}_{0}: X_{i, k} \sim f_{0}(x), \text { if } k<\lambda, \\
& \mathcal{H}_{1}: X_{i, k} \sim f_{1}(x), \text { if } k \geq \lambda .
\end{aligned}
$$

After sampling the observation signal $X_{i, k}$, during the $k^{\text {th }}$ time slot, the $i^{\text {th }}$ sensor $S_{i}$ calculates the log-likelihood ratio (LLR) $Z_{i, k}=\log \frac{f_{1}\left(X_{i, k}\right)}{f_{0}\left(X_{i, k}\right)}$. The LLR is then quantized to $q_{i, k}$ bits, forming a quantized message $U_{i, k}$, which is sent to the FC. This is accomplished by comparing the LLR with $2^{q_{i, k}}-1$ quantization thresholds. Without loss of generality, we assume that the quantized message $U_{i, k}$ takes values in the set $\left\{0,1, \ldots, 2^{q_{i, k}}-1\right\}$, corresponding to the $2^{q_{i, k}}$ quantization bins. At every time slot, the FC receives these quantized messages from the sensors which have enough energy for transmitting them successfully, and performs the cumulative sum (CUSUM) test for detecting a change in the distribution of the observation signal using a Cumulative Sum (CUSUM) based sequential detection algorithm as discussed in [19].

During each time slot, the fading channel gains between the sensors and the FC, denoted by $\left\{h_{i, k}\right\}, 1 \leq i \leq N, 1 \leq k \leq M$, are assumed to remain constant, but change from one time slot to another. We assume that these fading channel gains are statistically independent and identically distributed across time slots, as well as across the sensors. In this paper, we consider the case where each sensor decides to sense the observation signal, only if it has enough energy in the battery for sensing and sending its quantized information to the FC. Thus, $\nu_{i, k}$ is determined by the following rule:

$$
\nu_{i, k}= \begin{cases}1, & \text { if } B_{i, k} \geq E_{s}+q_{i, k} E_{i, k}^{b}, \\ 0, & \text { otherwise }\end{cases}
$$

where $E_{s}$ is the amount of energy required for sensing during each time slot and the battery state, the energy required for sending each quantized bits and the number of quantized bits at the $i^{\text {th }}$ sensor during the $k^{\text {th }}$ time slot are represented by $B_{i, k}, E_{i, k}^{b}$ and $q_{i, k}$, respectively. The amount of energy spent by the $i$-th sensor during that time slot, denoted by $E_{i, k}$, is then:

$$
E_{i, k}=\nu_{i, k}\left(E_{s}+q_{i, k} E_{i, k}^{b}\right) .
$$

Remark 1: It should be noted that we can also add an energy cost of processing the sample at the sensor including the energy required for computing the log-likelihood ratio and quantizing this ratio, to the sensing energy $E_{s}$. As long as this cost is constant across all time slots, the subsequent problem formulation remains unaltered, and essentially the maximum battery capacity can be thought of the original battery capacity minus the energy cost of signal processing at the sensor, or alternatively, $E_{s}$ can be thought of energy cost due to both sensing and signal processing.

If the maximum battery capacity of all sensors is assumed to be $B_{\max }$ and $H_{i, k}$ represents the amount of harvested energy of the $i^{\text {th }}$ sensor during the $k^{\text {th }}$ time slot (assuming that it can be stored and only used in the following time slot), then the battery dynamics can be expressed as follows:

$$
B_{i, k+1}=\min \left\{B_{\max }, B_{i, k}+H_{i, k}-E_{i, k}\right\} .
$$

We also assume that $H_{i, k}$ are i.i.d across the sensors and time slots.

Remark 2: It should be noted that the i.i.d. assumption for the channel gains and the harvested energies are only for convenience, and more general models such as finite state Markov chain models for both the channel gains and harvested energies can be considered with spatial correlation across the sensors. This will only affect the computations of the optimal policy for the causal case using the dynamic programming methodology, but not the development of the subsequent analysis.

If either a binary phase shift keying (BPSK) or a quadrature phase shift keying (QPSK) modulation technique is employed at the sensors, assuming fixed receiver noise power spectral density $N_{0}$ at the FC, the probability of error $P_{e}$ can be derived from the following expression [31] as a function of the energy required for transmitting each quantized bit $E_{i, k}^{b}$ :

$$
P_{e}=\frac{1}{2} \operatorname{erfc}\left\{\sqrt{h_{i, k} \frac{E_{i, k}^{b}}{N_{0}}}\right\}
$$

where erfc denotes the complementary error function.

Thus for a fixed required probability of error $P_{e}$, the energy required for transmitting each quantized bit can be written as:

$$
E_{i, k}^{b}=\frac{N_{0}}{h_{i, k}}\left\{\operatorname{erfc}^{-1}\left(2 P_{e}\right)\right\}^{2},
$$

We assume that the channel power gain $h_{i, k}$ and in turn the transmission energy for each quantized bit $E_{i, k}^{b}$ are computed at the FC by sending pilot symbols (from the FC to the sensors) and assuming channel reciprocity between the sensors and the FC. Thus, during the $k^{\text {th }}$ time slot, the battery state information $\mathbf{B}_{k}=\left\{B_{1, k}, B_{2, k} \ldots, B_{N, k}\right\}$, and the channel gains between the sensors and the $\mathrm{FC}, \mathbf{h}_{k}=$ $\left\{h_{1, k}, h_{2, k}, \ldots, h_{N, k}\right\}$, are available to the $\mathrm{FC}$ via feedback from the sensors via control channels.

\section{A. Quickest Change Detection at the FC}

In order to simplify the problem under investigation, we assume that the probability of error $P_{e}$ for the transmission of the quantized messages from the sensors to the FC is sufficiently small, so that the data sent by the active sensors are received at the FC with high probability and we ignore the effect of erroneous transmissions. As we will see shortly in the subsequent analysis, this amounts to scaling the received sum of the quantized LLR at the FC by $\left(1-P_{e}\right)$ when all sensors are required to satisfy identical $P_{e}$ for information transmission.

As the quantized message $U_{i, k}$ is discrete valued, we assume that $g_{i}^{j}$ denotes its the probability mass function (pmf), if the observations are drawn from the hypothesis $\mathcal{H}_{j}$. In order to quantize $Z_{i, k}$ to $q_{i, k}$ bits, we need to determine $2^{q_{i, k}}-1$ number of thresholds. Denoting the $l^{\text {th }}$ quantization threshold for the $i^{t h}$ sensor as $t_{l}^{i}$, the corresponding pmfs can then be expressed as follows:

$$
\begin{aligned}
& g_{i}^{1}(l)=\mathbb{F}_{1}\left(t_{l+1}^{i}\right)-\mathbb{F}_{1}\left(t_{l}^{i}\right), \\
& g_{i}^{0}(l)=\mathbb{F}_{0}\left(t_{l+1}^{i}\right)-\mathbb{F}_{0}\left(t_{l}^{i}\right),
\end{aligned}
$$


where $\mathbb{F}_{1}$ and $\mathbb{F}_{0}$ are the corresponding cumulative distribution functions for the probability distribution functions (pdf) $f_{1}$ and $f_{0}$, respectively. They can be determined as follows:

$$
\begin{aligned}
& \mathbb{F}_{1}(x)=\int_{-\infty}^{x} f_{1}(x) \mathrm{d} x, \\
& \mathbb{F}_{0}(x)=\int_{-\infty}^{x} f_{0}(x) \mathrm{d} x .
\end{aligned}
$$

Remark 3: For the special case when the pdfs are Gaussian distributed with varying mean but the same variance, the hypothesis testing problem becomes:

$$
\begin{aligned}
& \mathcal{H}_{0}: X_{i, k} \sim \mathcal{N}\left(0, \sigma^{2}\right), \text { if } k<\lambda \\
& \mathcal{H}_{1}: X_{i, k} \sim \mathcal{N}\left(\mu, \sigma^{2}\right), \text { if } k \geq \lambda
\end{aligned}
$$

where $\mu$ is the mean of the observation signal $X_{i, k}$ under the alternative hypothesis $\mathcal{H}_{1}$ and $\sigma^{2}$ is the variance of $X_{i, k}$ under both hypothesis $\mathcal{H}_{0}$ and $\mathcal{H}_{1}$. The corresponding LLR, $Z_{i, k}$, can be determined by the following expression [19]:

$$
Z_{i, k}=\log \frac{f_{1}\left(X_{i, k}\right)}{f_{0}\left(X_{i, k}\right)}=\frac{\mu X_{i, k}}{\sigma^{2}}-\frac{\mu^{2}}{2 \sigma^{2}} .
$$

The pmfs after quantization become:

$$
\begin{aligned}
& g_{i}^{1}(l)=\Phi\left\{\frac{t_{l+1}^{i}-\mu}{\sigma}\right\}-\Phi\left\{\frac{t_{l}^{i}-\mu}{\sigma}\right\} \\
& g_{i}^{0}(l)=\Phi\left\{\frac{t_{l+1}^{i}}{\sigma}\right\}-\Phi\left\{\frac{t_{l}^{i}}{\sigma}\right\}
\end{aligned}
$$

where $\Phi$ is the cumulative distribution function corresponding to the standard normal random variable.

After receiving the quantized message $U_{i, k}$ from the individual sensors, the FC computes the quantized LLR between hypotheses $\mathcal{H}_{1}$ and $\mathcal{H}_{0}$ as follows:

$$
Z^{q}(k)=\sum_{i=1}^{N} \log \frac{g_{i}^{1}\left(U_{i, k}\right)}{g_{i}^{0}\left(U_{i, k}\right)} .
$$

We should note that this quantized LLR $Z^{q}(k)$ is different from $Z_{i, k}$ which is the LLR computed at the individual sensors, and is used for computing the CUSUM test statistic at the FC. We denote $T$ as the stopping time, i.e., the time instant when the quickest change detection algorithm declares that a change has been detected in the distribution of the observation signal. We define the sensing strategy as $\boldsymbol{v}=\left\{\nu_{i, k} ; i=1, \ldots, N ; k=1, \ldots, M\right\}$, and quantization function as $\mathbf{q}=\left\{q_{i, k} ; i=1, \ldots, N ; k=1, \ldots, M\right\}$. The stopping time $T$ along with these parameters form the policy $\phi=(\boldsymbol{v}, \mathbf{q}, T)$.

The non-Bayesian quickest change detection focuses on detecting the change point as soon as possible after its actual occurrence. Thus the goal of this algorithm is to find the joint sensing and quantization policy $\phi$, that minimizes the average worst case detection delay (SADD) [24], which is defined as follows:

$$
S A D D(\phi)=\sup _{1 \leq \lambda \leq \infty} \mathbb{E}_{\lambda}(T-\lambda \mid T \geq \lambda)
$$

where $\mathbb{E}_{\lambda}$ denotes the expectation if the change occurs at time instant $\lambda$.

The purpose of this paper is to find an optimal sensing decision by $\boldsymbol{v}^{\star}$ and optimal quantization function by $\mathbf{q}^{\star}$, where we define the corresponding policy tuple as $\tilde{\phi}=\left(\boldsymbol{v}^{\star}, \mathbf{q}^{\star}, T\right)$. The optimal stopping time is then determined by the minimax change point detection procedure can be expressed as:

$$
\begin{array}{rl}
T^{\star}=\min _{T} & S A D D(\tilde{\phi}), \\
\text { s.t. } & \mathbb{E}_{\infty}[T]>\gamma ; \gamma>1 .
\end{array}
$$

In the above optimization problem $\mathbb{E}_{\infty}[T]$ denotes the expected stopping time decided by the algorithm when the change never occurs, i.e., $\lambda=\infty$. This quantity is termed as average run length to false alarm in the literature, because it represents the inverse of the rate of false alarm, which is lower bounded by the inverse of a low constant false alarm rate, i.e., the minimum expected duration to false alarm $\gamma>1$, where typically $\gamma$ is chosen to be large.

As mentioned earlier, it is well known that it is asymptotically (as the false alarm rate goes to zero) optimal [19] for the sensors to quantize their individual LLR to maximize their individual Kullback-Leibler divergence measure between the distributions after and before the change, when the FC employs a CUSUM based sequential change detection algorithm utilizing the total LLR based on quantized information received (at the FC) from all sensors. Therefore, the corresponding CUSUM test statistic at the FC is defined by the following recursion:

$$
W^{q}(k)=\max \left\{0, W^{q}(k-1)+Z^{q}(k)\right\}, W^{q}(0)=0 .
$$

Furthermore, the optimal stopping time for the CUSUM test can be obtained as:

$$
T^{\star}=\min \left\{k \geq 1: W^{q}(k) \geq r\right\}, r=\log \gamma
$$

For easier readability we have summarized the relevant parameters for our subsequent problem formulation in Table I (see top of next page).

\section{Optimization Problem Formulation}

The asymptotic performance (as $\gamma \rightarrow \infty$ ) of the optimal decentralized detection scheme using a CUSUM test operating with the total LLR at the FC for a fixed quantization strategy, when the nodes are sensing periodically at each sampling period [19] is as follows:

$$
S A D D(T) \sim \frac{\log \gamma}{\mathbf{I}_{\text {tot }}^{q}} \text { as } \gamma \rightarrow \infty,
$$

where $\mathbf{I}_{\text {tot }}^{q}$ is the total Kullback Leibler (KL) information number between the hypothesis $\mathcal{H}_{1}$ and $\mathcal{H}_{0}$, based on quantized information obtained from all the sensors. This performance limit is can be treated as a fundamental limit in decentralized detection in much the same way as the Cramer-Rao Bound is viewed as a fundamental limit in parameter estimation (see [30, p. 386]). Thus in a model-based setting, no other change detection algorithm (parametric or non-parametric) can achieve a smaller SADD subject to a vanishingly small probability of false alarm. 
TABLE I

SYSTEM PARAMETERS

\begin{tabular}{|c|c|}
\hline$X_{k}$ & Observation signal for $i^{t h}$ sensor during $k^{t h}$ time slot \\
$\nu_{i, k}$ & Sensing decision parameter for $i^{t h}$ sensor during $k^{t h}$ time slot \\
$Z_{i, k}$ & Log-likelihood ratio (LLR) for $i^{t h}$ sensor during $k^{t h}$ time slot \\
$q_{i, k}$ & Number of quantized bits sent to the FC from $i^{t h}$ sensor during $k^{t h}$ time slot \\
$U_{i, k}$ & Quantized message sent to FC from $i^{t h}$ sensor during $k^{t h}$ time slot \\
$h_{i, k}$ & Channel gain between $i^{t h}$ sensor and the FC during $k^{t h}$ time slot \\
$B_{i, k}$ & Battery state at the $i^{t h}$ sensor during the $k^{t h}$ time slot \\
$E_{s}$ & Energy required for sensing \\
$E_{i, k}^{b}$ & Energy required for transmitting one quantized bits from the $i^{t h}$ sensor during the $k^{t h}$ time slot \\
$B_{m a x}$ & Battery capacity \\
$H_{i, k}$ & Amount of energy harvested by the $i^{\text {th }}$ sensor during $k^{t h}$ time slot \\
$E_{i, k}$ & Energy usage by the $i^{t h}$ sensor during $k^{t h}$ time slot \\
$N_{0}$ & Noise power spectral density at the FC \\
$P_{e}$ & Probability of bit error at the FC \\
$g_{i}^{j}$ & Probability mass function, if the observation is drawn from hypothesis $\mathcal{H}_{j}$ \\
$t_{l}^{i}$ & $l^{t h}$ quantization threshold for $i^{t h}$ sensor \\
$\mu$ & Mean of $X_{i, k}$ under hypothesis $\mathcal{H}_{1}$ \\
$\sigma^{2}$ & Variance of $X_{i, k}$ under hypothesis $\mathcal{H}_{0}$ and $\mathcal{H}_{1}$ \\
$\lambda$ & Change point of the distribution \\
$R_{q_{i, k}}$ & The difference between KL divergence of unquantized and quantized distribution with $q_{i, k}$ quantization bits \\
$Z^{q}(k)$ & Sum of quantized LLRs received at FC from all the transmitting sensors \\
\hline
\end{tabular}

In the case of energy harvesting sensors, it is clear that for every given time slot, the number of active sensors depends on the corresponding battery states of the sensor, and is therefore random. An expression for $\mathbf{I}_{\text {tot }}^{q}$ can be determined for each time slot by summing the KL information measures for individual sensors over a given number of active sensors, assuming error-free transmission between the active sensors and the FC. As remarked earlier, we assume the probability of erroneous transmission, $P_{e}$, is sufficiently small so that we ignore its effect as essentially once $P_{e}$ is fixed and identical for all sensors, the total $\mathrm{KL}$ divergence measure at the FC is simply scaled by $\left(1-P_{e}\right)$ when transmission errors are taken into account. We denote the number of active users in the $k^{\text {th }}$ time slot by $n_{k}$, which is a random variable dependent on the channel state information and harvested energy, for a given sensing and quantization strategy (which in turn determines the battery state at the sensors). In that case, $\mathbf{I}_{\text {tot }}^{q}$ (for the $k$-th time slot) can be expressed as:

$$
\mathbf{I}_{\text {tot }}^{q}=\sum_{i=1}^{n_{k}} I\left(g_{i}^{1}, g_{i}^{0}\right)=\sum_{i=1}^{n_{k}} \sum_{l=0}^{2^{q_{i, k}-1}} g_{i}^{1}(l) \log \frac{g_{i}^{1}(l)}{g_{i}^{0}(l)}
$$

where $\mathbf{I}\left(g_{i}^{1}, g_{i}^{0}\right)$ denotes the $\mathrm{KL}$ divergence between the probability mass functions $g_{i}^{1}$ and $g_{i}^{0}$ of $i^{\text {th }}$ sensor $S_{i}$.

Minimizing the asymptotic average worst case detection delay is equivalent to the problem of maximizing $\mathbf{I}_{t o t}^{q}$, when the number of active sensors $n_{k}$, the channel gain $\mathbf{h}_{k}$ and the battery state $\mathbf{B}_{k}$ are deterministic and identical for all time slots. But the obvious limitation of such an approach is that it does not work when $n_{k}, \mathbf{h}_{k}, \mathbf{B}_{k}$ are varying randomly from one time slot to another. Thus, we are interested in optimizing the sensing and quantization policy over a finite time horizon by taking into account the effects of varying number of active sensors, channel gains and energy harvesting in different sensors on the quickest change detection process.
Note that this optimization problem formulation also allows a more general energy harvesting and expenditure model than the binary model studied in [25]. For a stationary and ergodic energy harvesting and channel gain process, the corresponding objective function $\frac{1}{M} \sum_{k=1}^{M} \mathbf{I}_{\text {tot }}^{q}$ can be interpreted as the expected KL information measure when the number of time slots (horizon length) $M \rightarrow \infty$. It should be noted that while the asymptotic upper bound on the average detection delay is not exactly inversely proportional to the average KL measure, the inverse of the average KL measure still provides a lower bound on the asymptotic average detection delay from Jensen's inequality. Additionally, the problem of maximizing the average KL measure can provide further insight into the optimal sensing and quantization strategies for minimizing the average detection delay in the asymptotic case when the probability of false alarm goes to zero. Thus, the optimization problem is formulated as follows:

$$
\begin{aligned}
\max _{\nu_{i, k}, q_{i, k}} & \sum_{k=1}^{M} \mathbb{E}_{n_{k}}\left\{\sum_{i=1}^{n_{k}}\left\{\nu_{i, k} \sum_{l=0}^{2^{q_{i, k}}-1} g_{i}^{1}(l) \log \frac{g_{i}^{1}(l)}{g_{i}^{0}(l)}\right\}\right\} \\
\text { s.t. } & \nu_{i, k} \in\{0,1\} ; \forall i, k, \\
& q_{i, k} \in\left\{1, \ldots, Q_{\max }\right\} ; \forall i, k, \\
& E_{i, k} \leq B_{i, k} ; \forall i, k .
\end{aligned}
$$

Remark 4: In the optimization problem mentioned above, $Q_{\max }$ denotes the maximum number of quantization bits per sample available to each sensor, whereas equations (19), (20) and (21) refer to the binary choice of sensing decision $\nu_{i, k}$, the feasible set of number of quantization bits $q_{i, k}$ and the energy causality constraint, respectively.

For the sake of completeness, we analyze an alternative optimization problem in Section VII, which focuses on minimizing the average inverse KL measure over a finite horizon rather than maximizing the average KL measure itself. This alternative optimization provides a better lower bound on 
the asymptotic detection delay compared to maximizing the average KL measure.

\section{Non-Causal Optimization With Finite Battery}

In this section, we analyze the optimization problem under the assumption that the channel gains between the sensors and the FC and the battery state information are non-causally available. For most practical applications, this assumption does not hold. However, this provides a benchmark of performance for the more practical scenario, when the information is causally available. It should be noted that in this optimization problem, both optimization variables $\nu_{i, k}$ and $q_{i, k}$ are discrete valued. In order to tackle the combinatorial nature of this problem, it is solved by DP similar to the causal scenario, which will be discussed in Section VI.

Remark 5: It should be noted that for a fixed sensing and quantization policy and non-causal knowledge about $\left\{\mathbf{B}_{k}, \mathbf{h}_{k}\right\}$, where $k=1, \ldots, M$, the objective function in the above optimization problem becomes deterministic and thus the expectation operator in (18) can be removed.

At the $k^{t h}$ time slot, when the CSI and ESI are noncausally available, the corresponding sensing policy $\nu_{i, k}$, and number of quantization bits $q_{i, k}$ for $i=1,2, \ldots, N$, is determined offline using the DP algorithm. Once $\nu_{i, k}$ and $q_{i, k}$ are determined, one needs to find the optimal positions of the quantization thresholds for quantizing the LLR, $Z i, k$ for the $i$-th sensor. Lets assume that $\psi_{i, k}^{*}$ represents the quantization function that optimizes the problem (18)-(21). Hence, we are interested in finding the $2^{q_{i, k}}-1$ thresholds $t_{0}, t_{1}, \ldots, t_{2^{q_{i, k}}-1}$ as follows:

$$
\psi_{i, k}^{*}\left(X_{i, k}\right)=b_{i, k} \text { only if } t_{b_{i, k}}<Z_{i, k} \leq t_{b_{i, k}+1}
$$

where $b_{i, k} \in\left\{0,1, \ldots, 2^{q_{i, k}}-1\right\}$.

From the optimization problem (18)-(21), it is evident that the optimal policy entails finding the sensing decision, number of quantization bits and the corresponding thresholds jointly using the DP. This is prohibitively complex because of the exponential computational complexity of the DP algorithm in the state and action space. In order to keep the computations tractable, we obtain the thresholds non-adaptively, i.e., first finding the optimal sensing decision and the number of quantization bits by DP, while applying a set of quantization thresholds pre-computed corresponding to the number of quantization bits found by DP. These quantization thresholds are designed for each value of the number of quantization bits $q_{i, k} \in\left\{1,2, \ldots, Q_{\max }\right\}$ by simply maximizing the KL divergence measure for a given time slot, rather than by considering all the time slots in the finite horizon. In the next three subsections, we will describe three policies for obtaining the thresholds, (i) the optimal threshold quantization (which maximized the KL Divergence measure at each time slot), (ii) a mean value theorem (MVT) based asymptotically optimal threshold (when the number of quantization levels goes to infinity, i.e., $Q_{\max } \rightarrow \infty$ ), and (iii) a simple uniform quantization policy, respectively.

\section{A. Optimal Threshold Quantization}

The KL divergence for the $k$-th slot based on the quantized LLR from $n_{k}$ active sensors can be expressed as follows:

$$
\begin{array}{r}
\mathcal{F}\left(\left\{t_{l}^{i}: l \in\left\{0, \ldots, 2^{q_{i, k}}-1\right\}\right\}\right) \\
=\sum_{i=1}^{n_{k}} \sum_{l=0}^{2^{q_{i, k}}-1} g_{i}^{1}(l) \log \frac{g_{i}^{1}(l)}{g_{i}^{0}(l)},
\end{array}
$$

under the constraints that $\nu_{i, k}$ and $q_{i, k}$ satisfy (19), (20) and (21).

As the KLD contribution from each sensor is only dependent on its own quantization thresholds, we can consider maximizing the KLD sensor separately with respect to its own quantization thresholds. Therefore the optimal solution for the thresholds for the $i$-th sensor can be found by solving for $\frac{\partial \mathcal{F}_{i}}{\partial t^{(}()_{l}}=0$, where $\mathcal{F}_{i}=\sum_{l=0}^{2^{q_{i, k}}-1} g_{i}^{1}(l) \log \frac{g_{i}^{1}(l)}{g_{i}^{0}(l)}$. Furthermore, in the interest of simplifying notations, we drop the sensor index $i$ from the threshold notation $t_{l}^{i}$. It should be noted that only two consecutive summands in the above sum are functions of $t_{l}$ as mentioned in (9) and (10), i.e., for a given sensor only $g^{1}(l), g^{0}(l), g^{1}(l-1)$ and $g^{0}(l-1)$ depend on $t_{l}$. Hence, the gradient expression can be written as:

$$
\frac{\partial \mathcal{F}_{i}}{\partial t_{l}}=\frac{\partial}{\partial t_{l}}\left\{\mathcal{F}_{i}^{1}+\mathcal{F}_{i}^{2}\right\}
$$

where $\mathcal{F}_{i}^{1}=g^{1}(l-1) \log \frac{g^{1}(l-1)}{g^{0}(l-1)}$ and $\mathcal{F}_{i}^{2}=g^{1}(l) \log \frac{g^{1}(l)}{g^{0}(l)}$, and the individual gradients can be simplified as follows:

$$
\begin{aligned}
\frac{\partial \mathcal{F}_{i}^{1}}{\partial t_{l}}= & \frac{\partial g^{1}(l-1)}{\partial t_{l}} \log \frac{g^{1}(l-1)}{g^{0}(l-1)} \\
& +g^{0}(l-1) \frac{\partial}{\partial t_{l}}\left\{\frac{g^{1}(l-1)}{g^{0}(l-1)}\right\}, \\
\frac{\partial \mathcal{F}_{i}^{2}}{\partial t_{l}}= & \frac{\partial g^{1}(l)}{\partial t_{l}} \log \frac{g^{1}(l)}{g^{0}(l)}+g^{0}(l) \frac{\partial}{\partial t_{l}}\left\{\frac{g^{1}(l)}{g^{0}(l)}\right\} .
\end{aligned}
$$

Thus the optimal thresholds can be found by solving the following equations:

$$
\frac{\partial \mathcal{F}_{i}^{1}}{\partial t_{l}}+\frac{\partial \mathcal{F}_{i}^{2}}{\partial t_{l}}=0, l=0,1, \ldots, 2^{q_{i, k}}-1 .
$$

Remark 6: If the pdfs under the hypothesis testing problem are Gaussian distributed as mentioned in Remark 1 of Section II, these two gradients can be further simplified by replacing the individual gradients of the cumulative distribution functions as follows:

$$
\begin{aligned}
& \frac{\partial g^{1}(l-1)}{\partial t_{l}}=-\frac{\partial g^{1}(l)}{\partial t_{l}}=\frac{1}{\sigma \sqrt{2 \pi}} e^{-\frac{1}{2}\left\{\frac{t_{l}-\mu}{\sigma}\right\}^{2}}, \\
& \frac{\partial g^{0}(l-1)}{\partial t_{l}}=-\frac{\partial g^{0}(l)}{\partial t_{l}}=\frac{1}{\sigma \sqrt{2 \pi}} e^{-\frac{1}{2}\left\{\frac{t_{l}}{\sigma}\right\}^{2}} .
\end{aligned}
$$

With some algebraic manipulations, (26) reduces to:

$$
\begin{aligned}
& \frac{\partial g^{1}(l-1)}{\partial t_{l}}\left\{\log \left\{\frac{\frac{g^{1}(l-1)}{g^{0}(l-1)}}{\frac{g^{1}(l)}{g^{0}(l)}}\right\}\right\} \\
& =\frac{\partial g^{0}(l-1)}{\partial t_{l}}\left\{\frac{g^{1}(l-1)}{g^{0}(l-1)}-\frac{g^{1}(l)}{g^{0}(l)}\right\} .
\end{aligned}
$$


Using the results of (27) and (28) in (29), we obtain the following set of simultaneous nonlinear equations:

$$
e^{-\frac{1}{2 \sigma^{2}}\left(\mu^{2}-2 \mu t_{l}\right)}=\left\{\frac{\frac{g^{1}(l-1)}{g^{0}(l-1)}-\frac{g^{1}(l)}{g^{0}(l)}}{\log \left\{\frac{\frac{g^{1}(l-1)}{g^{0}(l-1)}}{\frac{g^{1}(l)}{g^{0}(l)}}\right\}}\right\}, l=1,2, \ldots, 2^{q_{i, k}} .
$$

As mentioned earlier, the non-adaptive optimal thresholds can be obtained by solving the above mentioned equation by a nonlinear solver once we have determined the optimal number of quantization bits by DP.

\section{B. MVT Based Asymptotically Optimal Threshold Quantizer}

If sufficient bandwidth and harvested energies are available at the sensors, they may use larger numbers of quantization bits. In this case, a suitable approximation can be achieved by assuming $q_{i, k} \rightarrow \infty$, such that $t_{l}-t_{l-1} \rightarrow 0$. In order to find that approximation, first we apply the mean value theorem (MVT) as in [32] to both the numerator and the denominator of (30).

We introduce the function $r\left(t_{l}\right) \triangleq \frac{g^{1}(l)}{g^{0}(l)}$. Then, the numerator of (30) can be written as:

$$
\frac{g^{1}(l-1)}{g^{0}(l-1)}-\frac{g^{1}(l)}{g^{0}(l)}=r\left(t_{l-1}\right)-r\left(t_{l}\right) .
$$

With the assumption of $t_{l}-t_{l-1} \rightarrow 0$ and using the MVT we can write:

$$
r\left(t_{l-1}\right)-r\left(t_{l}\right) \approx r^{\prime}\left(t_{l-1}\right)\left(t_{l-1}-t_{l}\right),
$$

where $r^{\prime}\left(t_{l}\right)$ denotes the first order derivative of $r\left(t_{l}\right)$ with respect to $t_{l}$.

Similarly, we introduce the function $s\left(t_{l}\right) \triangleq \log r\left(t_{l}\right)=$ $\log \frac{g^{1}(l)}{g^{0}(l)}$. Thus, the denominator of (30) can be written as:

$$
\log \left\{\frac{\frac{g^{1}(l-1)}{g^{0}(l-1)}}{\frac{g^{1}(l)}{g^{0}(l)}}\right\}=s\left(t_{l-1}\right)-s\left(t_{l}\right) .
$$

Applying the MVT on the above expression gives:

$$
s\left(t_{l-1}\right)-s\left(t_{l}\right) \approx s^{\prime}\left(t_{l-1}\right)\left(t_{l-1}-t_{l}\right) .
$$

Finally, (30) can be simplified by using (31) and (32) to obtain the approximate relationship

$$
e^{-\frac{1}{2 \sigma^{2}}\left(\mu^{2}-2 \mu t_{l}\right)}=\frac{r^{\prime}\left(t_{l-1}\right)}{s^{\prime}\left(t_{l-1}\right)}, l=1,2, \ldots, 2^{q_{i, k}} .
$$

The set of nonlinear equations (33) can be used for determining the quantization thresholds for the MVT based asymptotically optimal scenario.

Remark 7: When the pdfs are Gaussian distributed as mentioned in Section II, the expression for $s^{\prime}\left(t_{l-1}\right)$ and $r^{\prime}\left(t_{l-1}\right)$ are related as:

$$
s^{\prime}\left(t_{l-1}\right)=r^{\prime}\left(t_{l-1}\right) \frac{\Phi\left\{\frac{t_{l}}{\sigma}\right\}-\Phi\left\{\frac{t_{l-1}}{\sigma}\right\}}{\Phi\left\{\frac{t_{l}-\mu}{\sigma}\right\}-\Phi\left\{\frac{t_{l-1}-\mu}{\sigma}\right\}} .
$$

Replacing $s^{\prime}\left(t_{l-1}\right)$ from (33) to (34) we obtain the following set of nonlinear equations:

$$
e^{-\frac{1}{2 \sigma^{2}}\left(\mu^{2}-2 \mu t_{l}\right)}=\frac{\Phi\left\{\frac{t_{l}-\mu}{\sigma}\right\}-\Phi\left\{\frac{t_{l-1}-\mu}{\sigma}\right\}}{\Phi\left\{\frac{t_{l}}{\sigma}\right\}-\Phi\left\{\frac{t_{l-1}}{\sigma}\right\}} .
$$

The advantage of the above set of equations is that starting from the largest threshold, one can recursively compute the entire sequence of thresholds going backwards and thus simplifying the computations involved in obtaining the optimal thresholds.

\section{Uniform Threshold Quantizer}

For this quantization policy, we first choose an interval of significance in the distribution of $X_{i, k}$, where most of the probability mass is concentrated, and ignore any value of $X_{i, k}$ outside that interval, such as mean plus or minus three times the standard deviation for a given Gaussian distribution. Supposing the upper and lower bounds for this interval are represented by $v_{x}$ and $u_{x}$, respectively, and the observation signal is quantized with $q_{i, k}$ number of bits, then the corresponding number of quantization bins and thresholds are $2^{q_{i, k}}$ and $2^{q_{i, k}}-1$, respectively. The length of each quantization bin for this uniform policy in such a scenario is:

$$
\Delta=\frac{v_{x}-u_{x}}{2^{q_{i, k}}},
$$

and the corresponding $j^{\text {th }}$ threshold is located at:

$$
t_{j}=u_{x}+\Delta j
$$

It should be noted that the choice of upper and lower bound for the interval of significance in this uniform quantization strategy influences the performance of the corresponding detection policy. Thus, additional performance gains may be obtained reasonable by optimizing the KLD with respect to these two aforementioned limits. However, numerical results show that the performance benefits obtained are marginal and therefore we use a fixed choice for $u_{x}, v_{x}$, e.g., mean plus minus 3 times the standard deviation for a Gaussian distribution.

It should be noted that the optimal, asymptotically optimal and uniform threshold quantization policies can be used for the scenario involving both the non-causal and the causal CSI scenario to be described in Section VI.

\section{AsYmptotic QuANTIZATION ANALYSis}

In this section we will provide an asymptotic characterization of the difference between the unquantized and the quantized KL divergence for the optimal quantizer, as the number of quantization intervals goes to infinity. This analysis provides us with a lower bound on the gap between the KLD performance with the optimal threshold quantization policy and the true unquantized KLD per time slot. This, in turn, helps provide a benchmark for choosing practical quantization policies and the maximum number of quantization bits that are sufficient in real applications. For the analysis in this section, since we are only considering the KLD in a given 
time slot, we assume that the number of active sensors participating in sequential detection process is fixed. This analysis is based on asymptotic quantization theory originally pursued for quadratic distortion measures in [33], and later extended to non-distortion type measures such as the KL divergence in [34]. As the following discussion largely mirrors the analysis in [34], we simply provide a summary of the intermediate steps in arriving at the final lower bound expression.

\section{A. Asymptotic Optimal Threshold Quantization Bound}

Note that before quantization, the distribution functions of the observation signal are $f_{1}(x)$ and $f_{0}(x)$ under the hypothesis $\mathcal{H}_{1}$ and $\mathcal{H}_{0}$, respectively. After quantization, the probability mass functions are denoted by $g_{1}$ and $g_{0}$, respectively. Similar to the previous section, we drop the index $i$ in $t_{l}^{i}$ for readability. For asymptotic quantization, i.e., when the number of quantization bits $q_{i, k} \rightarrow \infty$, we are interested in finding an asymptotic expression for the distortion function, i.e., the difference between the unquantized and the quantized divergence is given by:

$$
R_{q_{i, k}}=D\left(f_{1} \| f_{0}\right)-D_{q_{i, k}}\left(g_{1} \| g_{0}\right)
$$

where the unquantized KL divergence $D\left(f_{1} \| f_{0}\right)$ is defined as:

$$
D\left(f_{1} \| f_{0}\right)=\int_{-\infty}^{\infty} f_{1}(x) \log \frac{f_{1}(x)}{f_{0}(x)} \mathrm{d} x,
$$

and the quantized KL divergence $D_{L}\left(g_{1} \| g_{0}\right)$ is defined as:

$$
D_{q_{i, k}}=\sum_{l=0}^{2^{q_{i, k}}-1} g_{1}(l) \log \frac{g_{1}(l)}{g_{0}(l)} .
$$

For the analysis in this section, we divide the observation region into $2^{q_{i, k}}$ quantization bins. We denote the scalar quantization operation by $Q=(S, C)$, which consists of the quantization points $C=\left\{c_{1}, \ldots, c_{2} q_{i, k}\right\}$ and quantization intervals $S=\left\{S_{1}, \ldots, S_{2^{q_{i . k}}}\right\}$. The quantization operation can be expressed as:

$$
Q(x)=c_{l}, \text { for } x \in S_{l} .
$$

For the quantizer $Q$, let $V_{l}=\int_{S_{l}} \mathrm{~d} x$ denote the volume of the $l^{\text {th }}$ quantization bin. The specific point density [35] of $Q$ is defined as:

$$
\zeta(x)=\frac{1}{2^{q_{i, k}} V_{l}}, \text { for } x \in S_{l} .
$$

The corresponding specific inertial profile function $m(x)$ is defined as [33]:

$$
m(x)=\frac{\int_{S_{l}}\left\|y-c_{l}\right\|^{2} \mathrm{~d} y}{V_{l}^{3}}, \text { for } x \in S_{l},
$$

whereas, the log-likelihood ratio (LLR) is defined as:

$$
Z(x)=\log \frac{f_{1}(x)}{f_{0}(x)} .
$$

Given these details, the asymptotic bound on the distortion measure between the unquantized and the quantized divergence from the results in [34], can be summarized as follows:

$$
\lim _{q_{i, k} \rightarrow \infty} 2^{2 q_{i, k}} R_{q_{i, k}}=\frac{1}{2} \int \frac{f_{1}(x) \mathcal{F}(x)}{\zeta^{2}(x)} \mathrm{d} x,
$$

where

$$
\mathcal{F}(x)=\nabla Z(x)^{T} m(x) \nabla Z(x),
$$

which is called the Fisher covariation profile. In order to find the corresponding bound for $R_{q_{i, k}}$ corresponding to the optimal quantizer, we use the discrimination-optimal point density function [35] as follows:

$$
\zeta^{d}(x)=\frac{\left[f_{1}(x) \mathcal{F}(x)\right]^{\frac{1}{3}}}{\int\left[f_{1}(y) \mathcal{F}(y)\right]^{\frac{1}{3}} \mathrm{~d} y} .
$$

The asymptotic distortion function with the optimal point density can be expressed as:

$$
R_{q_{i, k}} \approx \frac{1}{2^{2 q_{i, k}+1}}\left(\int\left[f_{1}(x) \mathcal{F}(x)\right]^{\frac{1}{3}} \mathrm{~d} x\right)^{3} .
$$

Remark 8: For the scenario, when the observations are generated from the Gaussian distribution as mentioned in (6), the expression for the $\log$-likelihood ratio $Z(x)$ is as follows:

$$
Z(x)=\log \frac{f_{1}(x)}{f_{0}(x)}=\frac{(2 x-\mu) \mu}{2 \sigma^{2}} .
$$

Thus, the corresponding gradient of the log-likelihood ratio can be simplified to:

$$
\nabla Z(x)=\frac{\mu}{\sigma^{2}} .
$$

For the scalar quantizer, when the quantization point $c_{l}$ is represented by the midpoint of the quantization bin $S_{l}$, the value of the specific inertial profile function [35] is $m(x)=\frac{1}{12}$. Thus, the corresponding Fisher covariation profile function can be simplified to:

$$
\mathcal{F}(x)=\{\nabla Z(x)\}^{2} m(x)=\frac{1}{12} \frac{\mu^{2}}{\sigma^{4}} .
$$

Using the discrimination-optimal point density, we obtain the following asymptotic distortion function as in (43):

$$
R_{q_{i, k}} \approx \frac{1}{2^{2 q_{i, k}+1}}\left(\int f_{1}^{\frac{1}{3}}(x) \mathcal{F}^{\frac{1}{3}}(x) \mathrm{d} x\right)^{3},
$$

which is further simplified to:

$$
R_{q_{i, k}} \approx \frac{1}{12} \frac{\mu^{2}}{\sigma^{4}} \frac{1}{2^{2 q_{i, k}+1}}\left(\int\left(\frac{1}{\sigma \sqrt{2 \pi}}\right)^{\frac{1}{3}} e^{-\frac{(x-\mu)^{2}}{6 \sigma^{2}}} \mathrm{~d} x\right)^{3} .
$$

By substitution, the expression can be reduced to:

$$
R_{q_{i, k}} \approx \frac{1}{12} \frac{\mu^{2}}{\sigma^{4}} \frac{1}{2^{2 q_{i, k}+1}} \frac{1}{\sigma \sqrt{2 \pi}}\left(\sigma \sqrt{6} \int_{-\infty}^{\infty} e^{-z^{2}} \mathrm{~d} z\right)^{3} .
$$

Utilizing the standard integral $\int_{-\infty}^{\infty} e^{-a x^{2}} \mathrm{~d} x=\sqrt{\frac{\pi}{a}}$, for $a>0$, the asymptotic distortion function is evaluated to be:

$$
R_{q_{i, k}} \approx \frac{\sqrt{3} \pi \mu^{2}}{4 \sigma^{2}} \frac{1}{2^{2 q_{i, k}}} .
$$




\section{Causal Optimization With Finite Battery}

In this section, we consider the scenario involving causal channel and energy state information. For most practical applications, this is the more realistic scenario compared to its non-causal counterpart. The optimization problem in such a scenario can be formulated as a stochastic control problem which is usually solved using the dynamic programming (DP) algorithm.

\section{A. Information Pattern}

As described in the System Model section, the causal channel and energy state information is collected by the FC using channel estimation involving pilot symbols, and feedback from the sensors via control channels at the beginning of each slot. The information during the $k^{t h}$ time slot is recursively represented by $\mathbf{J}_{k}=\left\{\mathbf{h}_{k}, \mathbf{B}_{k}, \mathbf{J}_{k-1}\right\}$. It should be noted that for the purpose of simulation, we have ignored the amount of energy required for the above mentioned channel estimation/feedback process. If the energy required for this process is non negligible but fixed during each time slot, then the algorithm presented in the following section can be simply modified by subtracting that fixed amount of energy from the available energy in the battery and apply the DP algorithm with modified energy state information.

\section{B. Dynamic Programming Algorithm}

The stochastic control problem with causal information for optimizing the sensing decision and the number of quantization bits is analyzed in this subsection with the assumption that all sensors are capable of harvesting energy and storing it in a finite sized battery. The instantaneous KL divergence measure for the $k^{\text {th }}$ time slot can be expressed as follows:

$$
D\left(\boldsymbol{v}_{k}, \mathbf{q}_{k}\right)=\sum_{i=1}^{N}\left\{\nu_{i, k} \sum_{l=0}^{2^{q_{i}, k}-1} g_{i}^{1}(l) \log \frac{g_{i}^{1}(l)}{g_{i}^{0}(l)}\right\}
$$

where $\boldsymbol{v}_{k}=\left\{\nu_{1, k}, \nu_{2, k}, \ldots, \nu_{N, k}\right\}^{T}$ and $\mathbf{q}_{\mathbf{k}}=$ $\left\{q_{1, k}, q_{2, k}, \ldots, q_{N, k}\right\}^{T}$ denote the vector of sensing parameters $\nu_{i, k}$ and number of quantization bits $q_{i, k}$, respectively. For the optimization problem under consideration, we define the feasible set of optimization variables as $S=\left\{\left(\boldsymbol{v}_{k}, \mathbf{q}_{k}\right): \boldsymbol{v}_{k}, \mathbf{q}_{k}\right.$ satisfy (19), (20), (21) $\}$. With the objective of maximizing the expected sum of the KL divergence measure over a finite horizon, the following theorem presents the solution to the optimal sequences of decision to sense and number of quantization bits $\left(\left\{\nu_{i, k}\right\},\left\{q_{i, k}\right\}\right)$ using a DP algorithm:

Theorem 1: The value function of the finite horizon optimization problem for the finite battery scenario with initial causal information $\mathbf{J}_{1}=\left\{\mathbf{h}_{1}, \mathbf{B}_{1}\right\}, V_{1}\left(\mathbf{h}_{1}, \mathbf{B}_{1}\right)$ can be computed by the backward Bellman dynamic programming equation as follows:

$$
\begin{aligned}
V_{k}\left(\mathbf{h}_{\mathbf{k}}, \mathbf{B}_{\mathbf{k}}\right)=\max _{\left(\boldsymbol{v}_{k}, \mathbf{q}_{k}\right) \in S}\{ & D\left(\boldsymbol{v}_{k}, \mathbf{q}_{k}\right) \\
& \left.+\mathbb{E}\left\{V_{k+1}\left(\mathbf{h}_{k+1}, \mathbf{B}_{k+1} \mid \mathbf{h}_{k}, \mathbf{B}_{k}, \boldsymbol{v}_{k}, \mathbf{q}_{k}\right)\right\}\right\},
\end{aligned}
$$

for $1 \leq k \leq M-1$. It should be noted that the expectation is computed over the random variables $\mathbf{h}$ and $\mathbf{B}$ and the terminal
Algorithm 1 Dynamic Programming Algorithm for Calculation of Optimal Parameters

1: Initialization: The initial KL divergence $M^{\text {th }}$ time slot $D\left(\boldsymbol{v}_{M}, \mathbf{q}_{M}\right)$ is determined by (51).

2: Feasible Set is defined as $S=$ $\left\{\left(\boldsymbol{v}_{k}, \mathbf{q}_{k}\right): \boldsymbol{v}_{k}, \mathbf{q}_{k}\right.$ satisfy $\left.(19),(20),(21)\right\}$.

3: Value function for the $M^{\text {th }}$ time slot $V_{M}\left(\mathbf{h}_{M}, \mathbf{B}_{M}\right)=$ $\max _{\boldsymbol{v}_{M}, \mathbf{q}_{M} \in S} D\left(\boldsymbol{v}_{M}, \mathbf{q}_{M}\right)$.

4: for $k=M-1$ to 0 do

5: Value function for $k^{\text {th }}$ time slot:

$$
\begin{aligned}
V_{k}\left(\mathbf{h}_{\mathbf{k}}, \mathbf{B}_{\mathbf{k}}\right)= & \max _{\left(\boldsymbol{v}_{k}, \mathbf{q}_{k}\right) \in S}\left\{D\left(\boldsymbol{v}_{k}, \mathbf{q}_{k}\right)\right. \\
& \left.+\mathbb{E}\left\{V_{k+1}\left(\mathbf{h}_{k+1}, \mathbf{B}_{k+1} \mid \mathbf{h}_{k}, \mathbf{B}_{k}, \boldsymbol{v}_{k}, \mathbf{q}_{k}\right)\right\}\right\},
\end{aligned}
$$

where the inner expectation is computed numerically.

6: Optimal $\boldsymbol{v}_{k}$ and $\mathbf{q}_{k}$ can be computed by solving the following equation:

$$
\begin{aligned}
\left\{\boldsymbol{v}_{k}^{\star}, \mathbf{q}_{k}^{\star}\right\}= & \operatorname{argmax}_{\left(\boldsymbol{v}_{k}, \mathbf{q}_{k}\right) \in S}\left\{D\left(\boldsymbol{v}_{k}, \mathbf{q}_{k}\right)\right. \\
& \left.+\mathbb{E}\left\{V_{k+1}\left(\mathbf{h}_{k+1}, \mathbf{B}_{k+1} \mid \mathbf{h}_{k}, \mathbf{B}_{k}, \boldsymbol{v}_{k}, \mathbf{q}_{k}\right)\right\}\right\}
\end{aligned}
$$

7: end for

condition for the algorithm is given by:

$$
V_{M}\left(\mathbf{h}_{M}, \mathbf{B}_{M}\right)=\max _{\boldsymbol{v}_{M}, \mathbf{q}_{M} \in S} D\left(\boldsymbol{v}_{M}, \mathbf{q}_{M}\right) .
$$

Proof: The proof is standard and follows from the principle of optimality for finite horizon stochastic control problems [36].

The optimal values $\boldsymbol{v}_{k}^{\star}, \mathbf{q}_{k}^{\star}$ can be computed numerically by searching over the discretized values of optimization variables in the feasible set $S$ as follows:

$$
\begin{aligned}
\left\{\boldsymbol{v}_{k}^{\star}, \mathbf{q}_{k}^{\star}\right\}=\underset{\left(\boldsymbol{v}_{k}, \mathbf{q}_{k}\right) \in S}{\operatorname{argmax}} & \left\{D\left(\boldsymbol{v}_{k}, \mathbf{q}_{k}\right)\right. \\
& \left.+\mathbb{E}\left\{V_{k+1}\left(\mathbf{h}_{k+1}, \mathbf{B}_{k+1} \mid \mathbf{h}_{k}, \mathbf{B}_{k}, \boldsymbol{v}_{k}, \mathbf{q}_{k}\right)\right\}\right\}
\end{aligned}
$$

It should be noted that the above mentioned procedure is performed purely offline based on the statistical information available to the FC about the channel gain and the battery states at individual sensors. Based on this information, the FC creates a look up table for the optimal sensing decision $\nu_{i, k}^{\star}$ and the number of quantization bits $q_{i, k}^{\star}$ corresponding to the discretized values of channel gain and battery state. In practice, the FC receives the causal energy and channel state information in real time and finds the optimal values of the above mentioned optimization variables from the look up table corresponding to the closest discretized level of channel gain and battery state. Furthermore, these optimal values are subsequently shared with the individual sensors through an error free broadcast feedback channel.

We have summarized the above mentioned optimization procedure in an algorithmic form (see Algorithm 1) for computing the optimal sequences of sensing decision parameters $\left\{\boldsymbol{v}_{k}\right\}$ and number of quantization bits $\left\{\mathbf{q}_{k}\right\}$. 


\section{Alternative Optimization Problem}

In this subsection, we propose an alternative objective function for minimizing the average detection delay over a finite horizon of $M$ time slots. As mentioned in Section III, instead of maximizing $\frac{1}{M} \sum_{k=1}^{M} \mathbb{E}\left\{I_{\text {tot }}^{q}\right\}$, we choose to minimize the objective function $\frac{1}{M} \sum_{k=1}^{M} \mathbb{E}\left\{\frac{1}{I_{\text {tot }}^{q}}\right\}$ in this scenario. We can justify this choice by using Jensen's inequality, which says that for a convex function $f(x)$, the following inequality holds:

$$
\mathbb{E}(f(x)) \geq f(\mathbb{E}(x)) .
$$

Since $f(x)=x^{-1}$, where $x>0$, is convex, we can apply Jensen's inequality to obtain:

$$
\mathbb{E}\left\{\frac{1}{x}\right\} \geq \frac{1}{\mathbb{E}(x)}
$$

Applying the above to the $\mathrm{KL}$ divergence based cost $D\left(\boldsymbol{v}_{k}, \mathbf{q}_{k}\right)$, we have

$$
\mathbb{E}\left\{\frac{1}{D\left(\boldsymbol{v}_{k}, \mathbf{q}_{k}\right)}\right\} \geq \frac{1}{\mathbb{E}\left(D\left(\boldsymbol{v}_{k}, \mathbf{q}_{k}\right)\right)}
$$

Finally, applying Jensen's inequality again, we can show that

$$
\begin{aligned}
\frac{1}{M} \sum_{k=1}^{M} \mathbb{E}\left\{\frac{1}{D\left(\boldsymbol{v}_{k}, \mathbf{q}_{k}\right)}\right\} & \geq \frac{1}{M} \sum_{k=1}^{M} \frac{1}{\mathbb{E}\left(D\left(\boldsymbol{v}_{k}, \mathbf{q}_{k}\right)\right)} \\
& \geq \frac{1}{\frac{1}{M} \sum_{k=1}^{M} \mathbb{E}\left(D\left(\boldsymbol{v}_{k}, \mathbf{q}_{k}\right)\right)}
\end{aligned}
$$

This illustrates that in the optimization setting of the previous section (both causal and non-causal), we were minimizing a lower bound (by maximizing the denominator on the right hand side of the second inequality above) on the asymptotic expected detection delay, which is actually given by the left hand side of the first inequality above. Thus we consider the following optimization problem, which is expressed as:

$$
\begin{aligned}
\min _{\nu_{i, k}, q_{i, k}} & \sum_{k=1}^{M} \mathbb{E}_{n_{k}}\left\{\frac{1}{\sum_{i=1}^{n_{k}} \nu_{i, k} \sum_{l=0}^{2^{q_{i, k}}-1} g_{i}^{1}(l) \log \frac{g_{i}^{1}(l)}{g_{i}^{0}(l)}}\right\} \\
\text { s.t. } & \nu_{i, k} \in\{0,1\} ; \forall i, k, \\
& q_{i, k} \in\left\{1, \ldots, Q_{\max }\right\} ; \forall i, k \\
& E_{i, k} \leq B_{i, k} ; \forall i, k .
\end{aligned}
$$

Similar to the previous optimization problem, the optimal sensing policy and number of quantization bits for both noncausal and causal CSI are determined using the dynamic programming algorithm. Once the optimal number of quantization bits is known, the quantization thresholds are found using the same set of nonlinear equations as (30). This is due to the fact that, the threshold computation in Section IV-A, focuses on each individual sensor during each time slot in a decentralized fashion. Thus, maximization of $I\left(g^{1}, g^{0}\right)$ is equivalent to the problem of minimizing $\frac{1}{I\left(g^{1}, g^{0}\right)}$.

\section{Simulation Results}

In this section, we present a set of simulation results for both non-causal and causal information scenarios with finite battery capacity for both optimal and uniform quantization

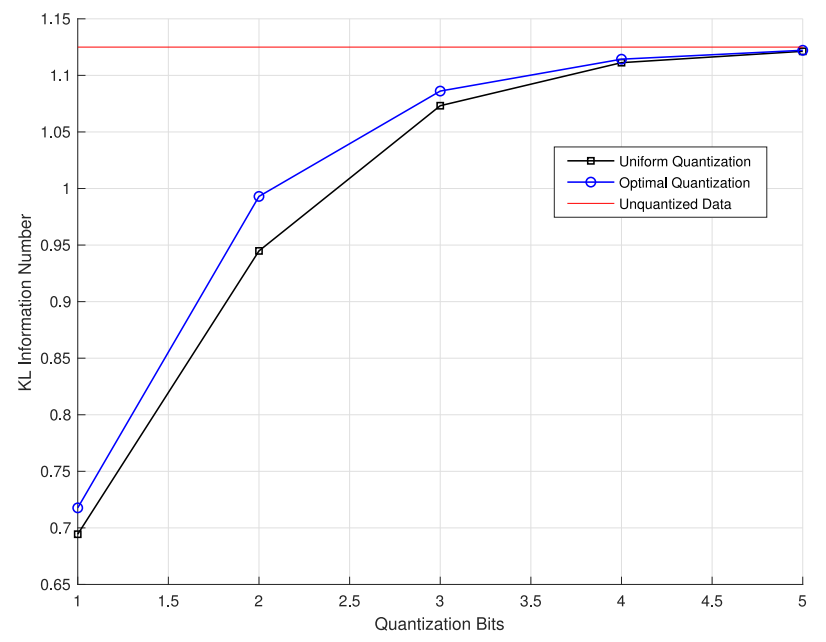

Fig. 2. Single slot single user KL information number for optimal, uniform quantization and unquantized policy as a function of number of quantization bits $q_{i, k}$.

policies. The power gain for the channel between the $i^{t h}$ sensor $S_{i}$ and the FC for the $k^{\text {th }}$ time slot, $h_{i, k}$, is modeled as an exponentially distributed random variable with unity mean. The amount of harvested energy, $H_{i, k}$, for the sensor $S_{i}$ during the $k$-th time slot is also assumed to be an exponentially distributed random variable with a mean of $1 \mu \mathrm{J}$. For the following simulations, we have assumed that the observations are generated from a Gaussian distribution as shown in (6) and the mean and the variance of the Gaussian distribution under hypothesis $\mathcal{H}_{1}$ are $\mu=1.5$ and $\sigma^{2}=1$, respectively. The probability of bit error for the quantized observation transmission to the $\mathrm{FC}$ is $P_{e}=0.005$. The noise power spectral density is assumed to be $N_{0}=0.02 \mu \mathrm{W} / \mathrm{Hz}$. The amount of energy required for sensing the observation signal is assumed to be $E_{s}=0.1 \mu \mathrm{J}$. The number of sensors are taken to be $N=2$. The maximum number of quantization bits is taken to be $Q_{\max }=5$. The choice of the value of $Q_{\max }$ is motivated by Fig. 2, which clearly shows that the KL divergence measure corresponding to both the optimal and uniform quantization policies and the unquantized case become virtually equal when $q_{i, k} \geq 5$. The initial battery level for all sensors are assumed to be $0.4 \mu \mathrm{J}$. For the DP algorithm implementation, the channel power gain $h_{i, k}$ and the battery state $B_{i, k}$ are both quantized to 4 discrete levels. The average of detection delay of change point is computed over $10^{4}$ of Monte-Carlo iterations for Fig. 3-Fig. 7.

For the plots in Fig. 3-Fig. 6, two policies of uniform and optimal quantization are taken into consideration. It is noteworthy that we have also proposed an MVT based asymptotically optimal quantizer in Section VI-B. For the sake of comparison, we include the one slot KL information number for all three quantization strategies in Table IIa, where we only filled the KL information values for the MVT based asymptotically optimal quantizer, when the number of quantization bits is significantly high. This is due to the fact that for the above mentioned quantizer, the asymptotic assumption of $t_{l}-t_{l-1} \rightarrow 0$ does not hold when the number of quantization bits is small, which results in significant inaccuracy in 


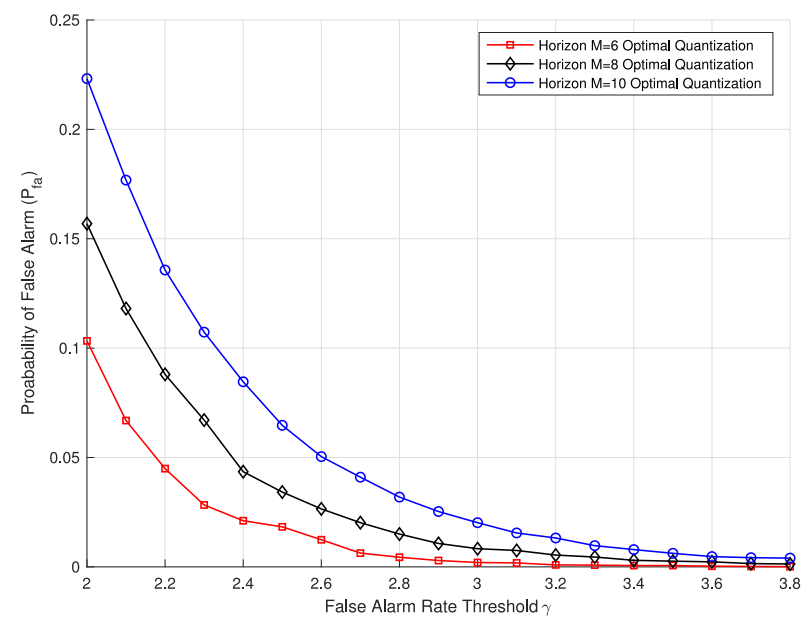

Fig. 3. Probability of false alarm $P_{f a}$ vs false alarm rate bound $\gamma$ for noncausal CSI with optimal quantization policy for Horizon length $M=6,8,10$.

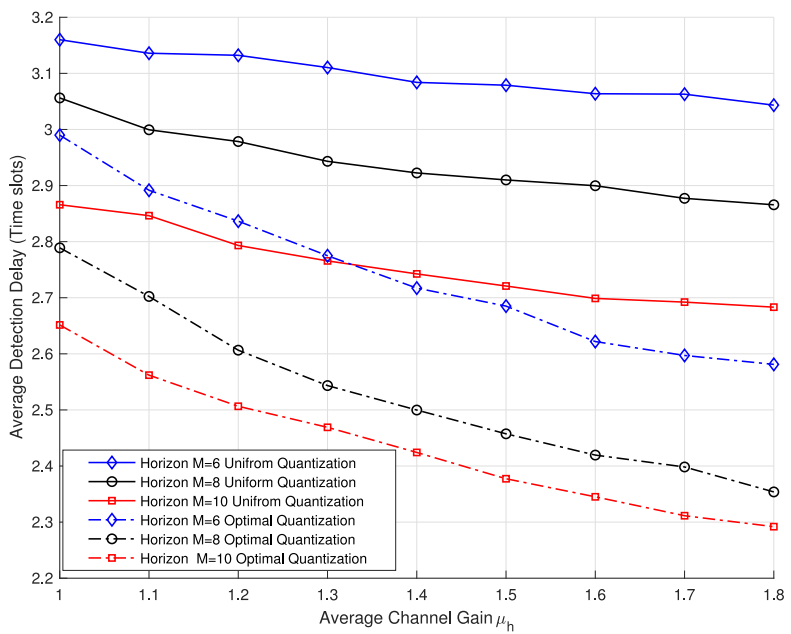

Fig. 4. Average detection delay (Time slots) vs mean channel gain $\mu_{h}$ for non-causal CSI with optimal and uniform quantization policy for horizon length $M=6,8,10$.

determining the thresholds. Thus, it performs poorly compared to its other two counterparts, especially when the number of quantization bits is small. However, when we increase the number of quantization bits significantly, then the MVT based asymptotically optimal quantization strategy performs similar to the other two policies.

Fig. 2 shows the comparative performance of the KL information number for the single sensor and one time slot case. We have plotted the KL information number for the unquantized observation, optimal, and uniform quantization policies with respect to varying number of quantization bits. The KL information number of the unquantized observation case can be easily shown to be $\frac{\mu^{2}}{2 \sigma^{2}}$. This signifies an upper bound for the quantized observation scenarios. As expected, Fig. 2 shows that the optimal quantization policy is superior to its uniform counterpart. It is also noticeable that the KL information number for all three policies become comparable as the number of quantization bits increases. In Table IIa we illustrate the difference of the KL information number of

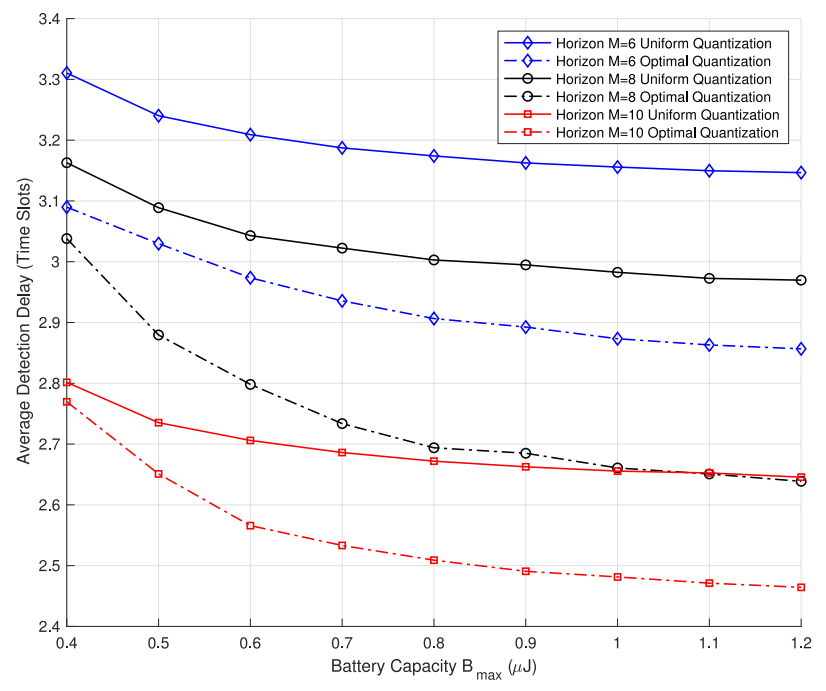

Fig. 5. Average detection delay (Time slots) vs Battery capacity $B_{\max }$ for non-causal CSI with optimal and uniform quantization policy for horizon length $M=6,8,10$.

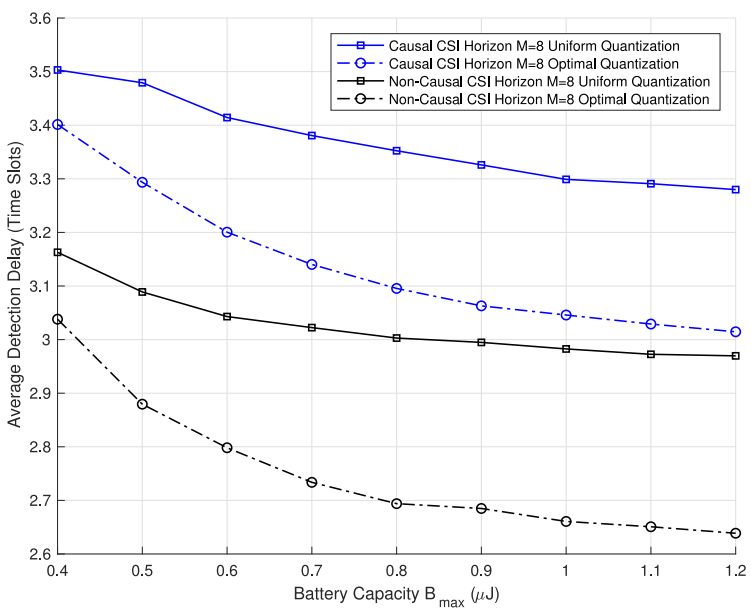

Fig. 6. Average detection delay (Time slots) vs Battery capacity $B_{\max }$ for non-causal and causal CSI with optimal and uniform quantization policy for horizon length $M=8$, and $\mu_{h}=1$.

the unquantized observation scenario and the optimal quantization strategy. In Table IIb, the aforementioned difference obtained by the simulation is compared with the asymptotic analytic bound introduced in Section V. Similar to Table IIa, it is expected that the asymptotic approximation does not hold when the number of quantization bits is low. Thus, we have only included the aforementioned difference in the asymptotic analytic bound column, when the number of quantization bits is sufficiently high, which is in clear agreement with the numerically evaluated results for the optimal quantization case.

Fig. 3 shows the probability of false alarm $P_{f a}$ with respect to varying levels of the false alarm rate lower bound parameter $\gamma$ for the non-causal CSI scenario and horizon length $M=6,8,10$ for the optimal quantization policy. We notice that the probability of false alarm increases with increasing horizon length for small values of $\gamma$. This is due to the fact that average detection delay decreases with increasing horizon length, which in turn effectively decreases the expected 


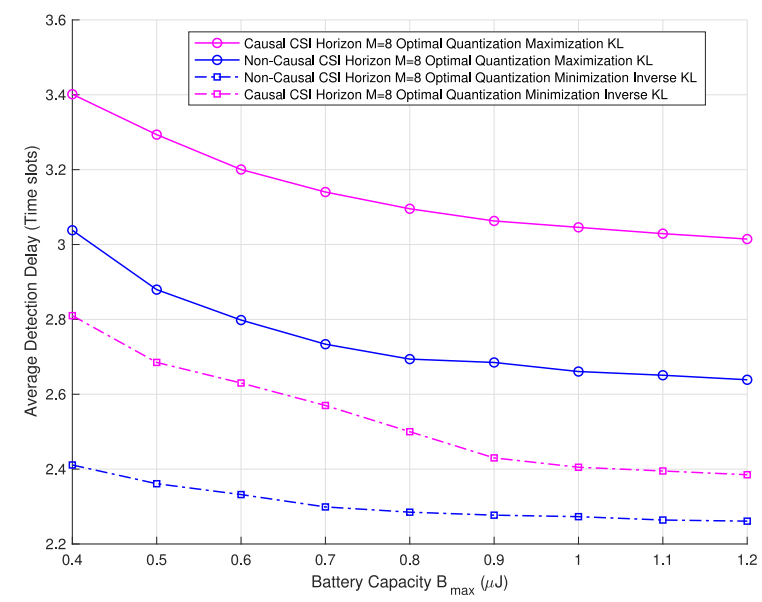

Fig. 7. Average detection delay (Time slots) vs Battery capacity $B_{\max }$ for non-causal and causal CSI for optimal quantization policy two different objective functions for horizon length $M=8$, and $\mu_{h}=1$.

TABLE II

(a) KL INFORMATION NUMBER COMPARISON. (b) KL INFORMATION NUMBER DiFFERENCE

(a)

\begin{tabular}{|l|c|c|c|}
\hline$q_{i, k}$ & MVT Optimal & Uniform & Optimal \\
\hline 3 & - & 1.0732 & 1.0861 \\
\hline 4 & - & 1.112 & 1.1143 \\
\hline 5 & - & 1.1213 & 1.1222 \\
\hline 6 & - & 1.1239 & 1.1245 \\
\hline 7 & 0.3146 & 1.1245 & 1.1247 \\
\hline 8 & 0.6831 & 1.1247 & 1.1248 \\
\hline 9 & 1.0834 & 1.1248 & 1.1248 \\
\hline
\end{tabular}

(b)

\begin{tabular}{|l|c|c|}
\hline$q_{i, k}$ & Optimal & Asymptotic Analytic Bound \\
\hline 1 & 0.4073 & - \\
\hline 2 & 0.1320 & - \\
\hline 3 & 0.0389 & - \\
\hline 4 & 0.0107 & 0.0136 \\
\hline 5 & 0.0028 & 0.0032 \\
\hline 6 & 0.0005 & 0.0008 \\
\hline 7 & 0.0003 & 0.0002 \\
\hline 8 & 0.0002 & $4.7 \times 10^{-5}$ \\
\hline 9 & 0.0002 & $1.1 \times 10^{-5}$ \\
\hline
\end{tabular}

time to false alarm, thus resulting in an increase of the false alarm probability. For the remaining plots, we have fixed the probability of false alarm to be $P_{f a}=0.01$. The corresponding values of $\gamma$ are extracted from Fig. 3 and utilized for the simulations involving this specific scenario. Although not explicitly shown here, we have also used similar plots for the simulations involving uniform quantization for the non-causal CSI scenario and both optimal and uniform quantization for the causal CSI scenario.

In Fig. 4, we have plotted the average detection delay with respect to the average channel power gain parameter $\mu_{h}$, keeping the battery capacity fixed at $B_{\max }=0.8 \mu \mathrm{J}$ for the non-causal CSI scenario. We note that the average detection delay in the optimal quantization case decays faster than the corresponding uniform ones. Fig. 5 shows the plot of average detection delay with respect to the battery capacity $B_{\max }$ where the average channel gain $\mu_{h}=1$ for non-causal information. As for numerical comparison, we notice from
Fig. 5 that for $M=10$ and $B_{\max }=0.7 \mu \mathrm{J}$, the average detection delay corresponding to the uniform quantization policy is 5.9 percent higher as compared to its optimal counterpart. It should also be noted that the average detection delay decreases with increasing horizon length. This intuitively makes sense for the non-causal scenario, where an increase in horizon length signifies availability of more information before the transmission process and it helps the sensors to plan its quantization and sensing strategy and in turn their energy usage in a better way.

Fig. 6 is a comparative plot demonstrating the average detection delay with respect to the battery capacity $B_{\max }$ while keeping the mean channel gain $\mu_{h}=1$ for both the optimal and the uniform quantization with non-causal and causal CSI where the horizon length is taken to be $M=8$. As for the numerical comparison, it can be seen that for $B_{\max }=0.7 \mu \mathrm{J}$, the average detection delay corresponding to the optimal quantization policy for the causal CSI scenario is 15.3 percent higher as compared to its non-causal counterpart.

In Fig. 7, we have plotted the average detection delay with respect to the battery capacity $B_{\max }$ for horizon length $M=8$ and the mean channel gain $\mu_{h}=1$. The figure includes the plots for both the causal and the non-causal scenario with the optimal quantizer determined by two different objective functions corresponding to maximizing average sum of $\mathrm{KL}$ divergence measure and minimizing the average sum of the inverse of the KL divergence measure. Out of the two objective functions and corresponding optimization problems, the simulation shows that minimization of the inverse KL measure performs better than the maximization of the KL measure. As a numerical comparison, we see that for $B_{\max }=0.7 \mu \mathrm{J}$, the average detection delay corresponding to the optimal quantizer, which maximizes the expected sum KL measure, is 22 percent higher compared to the optimal quantizer which minimizes the expected sum of the inverse of KL measure for the causal CSI scenario. In Fig. 8, we have plotted the average detection delay with respect to the average channel gain for optimal quantization policy that minimizes the inverse of KL measure. The average detection delays are plotted for the horizon length $M=6,8,10$ in the non-causal CSI scenario while keeping the battery capacity fixed at $B_{\max }=0.8 \mu \mathrm{J}$. Similar to Fig. 4, we notice from Fig. 8, that the average detection delay decreases with increasing average channel gain and increasing horizon length.

As mentioned earlier, we notice from Fig. 7 and comparing Fig. 4 with Fig. 8, that the alternate optimal quantization policy performs better (in terms of the average detection delay) than the optimal quantization policy which maximizes the expected sum of the KL measure. However, from Fig. 9, where we compare the probability of false alarm rates for the two optimization problems, we notice that the performance improvement of the alternate optimal quantization policy comes at the expense of a higher probability of false alarm $P_{f a}$. We also notice from Fig. 4 and Fig. 8, that the decrease in the average detection delay due to increase in horizon length is more prominent for the first optimal quantization policy as compared to its alternate counterpart. As a 


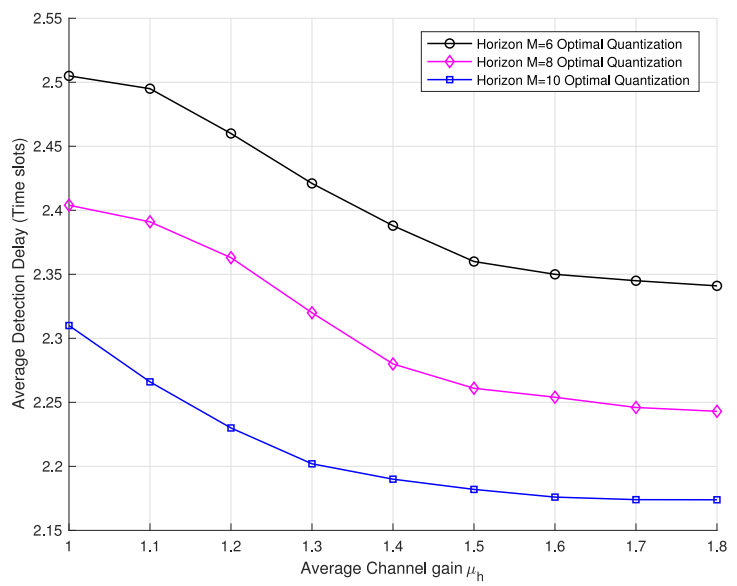

Fig. 8. Average detection delay (Time slots) vs mean channel gain $\mu_{h}$ for non-causal CSI for alternative optimal quantization policy with horizon length $M=6,8,10$.

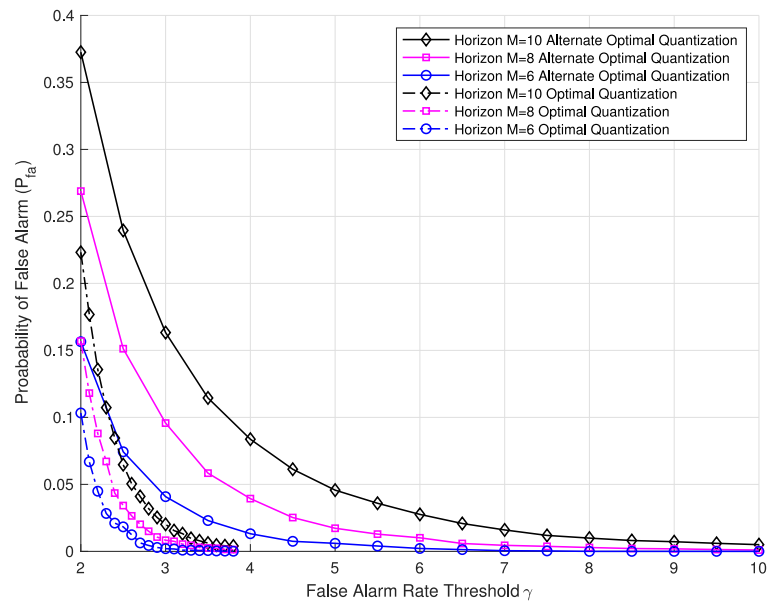

Fig. 9. Probability of false alarm $P_{f a}$ vs false alarm rate bound $\gamma$ for noncausal CSI with Optimal (expected KL maximizing) and Alternative optimal quantization (expected inverse KL minimizing) policy for Horizon length $M=6,8,10$

numerical comparison, for a mean channel gain $\mu_{h}=1$, the average detection delay for horizon length $M=8$ is 6.6 percent less compared to the scenario involving $M=6$, whereas for the alternative optimal quantization policy the corresponding decrease in average detection delay is 4 percent.

\section{CONCLUSION AND FUTURE WORK}

This paper investigated the problem of minimizing the average detection delay of a quickest change detection framework for the decentralised multi-sensor scenario where each sensor is capable of harvesting energies from its surroundings. Using the asymptotic result that expected detection delay is inversely proportional to the KL divergence measure between the densities of the observations after and before the change, we derived an optimal sensing and quantization strategy for such a problem by maximizing the expected sum of the KL divergence measure over a finite horizon, employing DP algorithms for both non-causal and causal scenarios. The non-causal CSI scenario provides a benchmark of average detection delay for the more realistic causal counterpart. We also derived closed form expression for the optimal thresholds for a fixed number quantization bits by maximizing the KL divergence measure for each time slot. This closed form expression is further simplified for the scenario when the number quantization bits is really high. We have also proposed a uniform quantization strategy as a heuristic policy. We have provided an analysis for the difference between the KL information measure for the unquantized and the quantized observation for the optimal strategy in the asymptotic case using asymptotic quantization theory. Simulation results indicate that the optimal quantizer significantly outperforms its uniform counterpart when the number of quantization bits is low. This performance benefit becomes marginal as the number of quantization bits increases. Future extensions of this work include formulation of optimization problems for the scenario when individual sensors perform the quickest change detection themselves and send local decisions to the FC, rather than sending quantized log-likelihood ratios. Other possible extensions of the work could be to the Bayesian framework, where the unknown change point is assumed to be a random process with a certain known probability distribution, or to quickest change detection of Generalized Likelihood Ratio (GLR) test, where the parameters of the distribution after the change are unknown. Extensions to change detection in non-parametric settings as considered in [20], [21] can also be formulated with energy harvesting sensors with window based sampling techniques, although there are non-trivial challenges concerning a suitable optimization problem formulation due to the absence of analytical expressions or bounds for the worst case average detection delay for such algorithms. Such extensions will thus be considered in future work.

\section{REFERENCES}

[1] V. C. Gungor and G. P. Hancke, "Industrial wireless sensor networks: Challenges, design principles, and technical approaches," IEEE Trans. Ind. Electron., vol. 56, no. 10, pp. 4258-4265, Oct. 2009.

[2] C.-Y. Chong and S. P. Kumar, "Sensor networks: Evolution, opportunities, and challenges," Proc. IEEE, vol. 91, no. 8, pp. 1247-1256, Aug. 2003.

[3] V. C. Gungor, B. Lu, and G. P. Hancke, "Opportunities and challenges of wireless sensor networks in smart grid," IEEE Trans. Ind. Electron., vol. 57, no. 10, pp. 3557-3564, Oct. 2010.

[4] I. F. Akyildiz, W. Su, Y. Sankarasubramaniam, and E. Cayirci, "A survey on sensor networks," IEEE Commun. Mag., vol. 40, no. 8, pp. 102-114, Aug. 2002.

[5] O. Ozel, J. Yang, and S. Ulukus, "Optimal broadcast scheduling for an energy harvesting rechargeable transmitter with a finite capacity battery," IEEE Trans. Wireless Commun., vol. 11, no. 6, pp. 2193-2203, Jun. 2012.

[6] O. Ozel and S. Ulukus, "Achieving AWGN capacity under stochastic energy harvesting," IEEE Trans. Inf. Theory, vol. 58, no. 10, pp. 6471-6483, Oct. 2012.

[7] K. Tutuncuoglu and A. Yener, "Optimum transmission policies for battery limited energy harvesting nodes," IEEE Trans. Wireless Commun., vol. 11, no. 3, pp. 1180-1189, Mar. 2012.

[8] H. A. Inan, D. Shaviv, and A. Özgür, "Capacity of the energy harvesting Gaussian MAC," IEEE Trans. Inf. Theory, vol. 64, no. 4, pp. 2347-2360, Apr. 2018.

[9] K. Tutuncuoglu and A. Yener, "Transmission policies for asymmetric interference channels with energy harvesting nodes," in Proc. 4th IEEE Int. Workshop Comput. Adv. Multi Sensor Adapt. Process. (CAMSAP), San Juan, Puerto Rico, Dec. 2011, pp. 197-200.

[10] N. Zlatanov, D. W. K. Ng, and R. Schober, "Capacity of the two-hop relay channel with wireless energy transfer from relay to source and energy transmission cost," IEEE Trans. Wireless Commun., vol. 16, no. 1, pp. 647-662, Jan. 2017. 
[11] B. Clerckx, R. Zhang, R. Schober, D. W. K. Ng, D. I. Kim, and H. V. Poor, "Fundamentals of wireless information and power transfer: From RF energy harvester models to signal and system designs," IEEE J. Sel. Areas Commun., vol. 37, no. 1, pp. 4-33, Jan. 2019.

[12] V. Sharma, U. Mukherji, V. Joseph, and S. Gupta, "Optimal energy management policies for energy harvesting sensor nodes," IEEE Trans. Wireless Commun., vol. 9, no. 4, pp. 1326-1336, Apr. 2010.

[13] Z. Ding, S. M. Perlaza, I. Esnaola, and H. V. Poor, "Power allocation strategies in energy harvesting wireless cooperative networks," IEEE Trans. Wireless Commun., vol. 13, no. 2, pp. 846-860, Feb. 2014

[14] M. Gregori and M. Payaro, "Energy-efficient transmission for wireless energy harvesting nodes," IEEE Trans. Wireless Commun., vol. 12, no. 3, pp. 1244-1254, Mar. 2013.

[15] X. Li, C. You, S. Andreev, Y. Gong, and K. Huang, "Wirelessly powered crowd sensing: Joint power transfer, sensing, compression, and transmission," IEEE J. Sel. Areas Commun., vol. 37, no. 2, pp. 391-406, Feb. 2019.

[16] R. Du, M. Xiao, and C. Fischione, "Optimal node deployment and energy provision for wirelessly powered sensor networks," IEEE J. Sel. Areas Commun., vol. 37, no. 2, pp. 407-423, Feb. 2019.

[17] S. Kay, Fundamentals of Statistical Signal Processing: Detection Theory (Prentice Hall Signal Processing Series). Englewood Cliffs, NJ, USA: Prentice-Hall, 1998.

[18] H. Poor and O. Hadjiliadis, Quickest Detection. Cambridge, U.K. Cambridge Univ. Press, 2008.

[19] A. G. Tartakovsky and V. V. Veeravalli, "Asymptotically optimal quickest change detection in distributed sensor systems," Sequential Anal., vol. 27, no. 4, pp. 441-475, 2008.

[20] M. Corneli, P. Latouche, and F. Rossi, "Multiple change points detection and clustering in dynamic networks," Stat. Comput., vol. 28, no. 5, pp. 989-1007, Sep. 2018.

[21] D.-H. Tran, "Change detection in streaming data," Ph.D. dissertation, Faculty Comput. Sci. Autom., Ilmenau Univ. Technol., Ilmenau, Germany, Oct. 2013.
[22] T. Banerjee and V. V. Veeravalli, "Bayesian quickest change detection under energy constraints," in Proc. Inf. Theory Appl. Workshop, Feb. 2011, pp. 1-10.

[23] G. Lorden, "Procedures for reacting to a change in distribution," Ann. Math. Stat., vol. 42, no. 6, pp. 1897-1908, Dec. 1971.

[24] M. Pollak, "Optimal detection of a change in distribution," Ann. Stat., vol. 13, no. 1, pp. 206-227, Mar. 1985.

[25] J. Geng and L. Lai, "Non-Bayesian quickest change detection with stochastic sample right constraints," IEEE Trans. Signal Process. vol. 61, no. 20, pp. 5090-5102, Oct. 2013.

[26] J. Geng, E. Bayraktar, and L. Lai, "Bayesian quickest change-point detection with sampling right constraints," IEEE Trans. Inf. Theory, vol. 60, no. 10 , pp. 6474-6490, Oct. 2014.

[27] G. V. Moustakides, "Decentralized CUSUM change detection," in Proc. 9th Int. Conf. Inf. Fusion, Florence, Italy, 2006, pp. 1-6.

[28] V. V. Veeravalli, "Decentralized quickest change detection," IEEE Trans. Inf. Theory, vol. 47, no. 4, pp. 1657-1665, May 2001.

[29] S. Biswas, S. Knorn, S. Dey, and A. Ahlen, "Quantized non-Bayesian quickest change detection with energy harvesting," in Proc. IEEE Glob. Commun. Conf. (GLOBECOM), Dec. 2018, pp. 1-7.

[30] A. G. Tartakovsky, I. Nikiforov, and M. Basseville, Sequential Analysis: Hypothesis Testing and Change Point Detection. Boca Raton, FL, USA: CRC Press, 2015.

[31] D. Tse and P. Viswanath, Fundamentals of Wireless Communication. Cambridge, U.K.: Cambridge Univ. Press, 2005.

[32] A. Khoshnevis and A. Sabharwal, "Performance of quantized power control in multiple antenna systems," in Proc. IEEE Int. Conf. Commun., vol. 2. Paris, France, Jun. 2004, pp. 803-807.

[33] A. Gersho, "Asymptotically optimal block quantization," IEEE Trans. Inf. Theory, vol. IT-25, no. 4, pp. 373-380, Jul. 1979.

[34] R. Gupta and A. O. Hero, "High-rate vector quantization for detection," IEEE Trans. Inf. Theory, vol. 49, no. 8, pp. 1951-1969, Aug. 2003.

[35] S. Na and D. L. Neuhoff, "Bennett's integral for vector quantizers," IEEE Trans. Inf. Theory, vol. 41, no. 4, pp. 886-900, Jul. 1995.

[36] D. P. Bertsekas, Dynamic Programming and Optimal Control, 2nd ed. Belmont, MA, USA: Athena Sci., 2000. 